\title{
Comorbidities Associated with Large Abdominal Aortic Aneurysms
}

\author{
Verena Müller, MD ${ }^{1, \#(~ M i l e n a ~ M i s z c z u k, ~ M D ~}{ }^{2, \#}$ Christian E. Althoff, MD 3 \\ Andrea Stroux, Dipl. Math. ${ }^{4 \odot}$ Andreas Greiner, Dipl. Math. ${ }^{2}$ Helena Kuivaniemi, MD, PhD ${ }^{5 \odot}$ \\ Irene Hinterseher, MD, $\mathrm{PhD}^{2 \odot}$
}

1 Surgical Clinic, Campus Charité Mitte and Campus Virchow-

Klinikum, Charité - Universitätsmedizin Berlin, corporate member of Freie Universität Berlin, Humboldt-Universität zu Berlin, and Berlin Institute of Health, Berlin, Germany

2 Vascular Surgery Clinic, Charité - Universitätsmedizin Berlin, corporate member of Freie Universität Berlin, Humboldt-Universität zu Berlin, and Berlin Institute of Health, Berlin, Germany

3 Institute of Radiology, Campus Charité Mitte, Charité Universitätsmedizin Berlin, corporate member of Freie Universität Berlin, Humboldt-Universität zu Berlin, and Berlin Institute of Health, Berlin, Germany

${ }^{4}$ Institute of Medical Biometrics and Clinical Epidemiology, Charité Universitätsmedizin Berlin, corporate member of Freie Universität Berlin, Humboldt-Universität zu Berlin, and Berlin Institute of Health, Berlin, Germany

${ }^{5}$ Division of Molecular Biology and Human Genetics, Department of Biomedical Sciences, Faculty of Medicine and Health Sciences, Stellenbosch University, Tygerberg, South Africa

\begin{abstract}
Address for correspondence Irene Hinterseher, MD, PhD, Vascular Surgery Clinic, Campus Charité Benjamin Franklin, Hindenburgdamm 30, 12200 Berlin, Germany (e-mail: irene.hinterseher@charite.de).
\end{abstract}

AORTA 2019;7:108-114.

\section{Abstract \\ Keywords \\ - abdominal aortic aneurysm \\ - comorbidities \\ - risk factor \\ - lung function \\ - smoking \\ - diverticulosis}

Background Abdominal aortic aneurysm has become increasingly important owing to demographic changes. Some other diseases, for example, cholecystolithiasis, chronic obstructive pulmonary disease, and hernias, seem to co-occur with abdominal aortic aneurysm. The aim of this retrospective analysis was to identify new comorbidities associated with abdominal aortic aneurysm.

Methods We compared 100 patients with abdominal aortic aneurysms and 100 control patients. Their preoperative computed tomographic scans were examined by two investigators independently, for the presence of hernias, diverticulosis, and cholecystolithiasis. Medical records were also reviewed. Statistical analysis was performed using univariate analysis and multiple logistic regression analysis.

Results The aneurysm group had a higher frequency of diverticulosis $(p=0.008)$. There was no significant difference in the occurrence of hernia $(p=0.073)$ or cholecystolithiasis $(p=1.00)$. Aneurysm patients had a significantly higher American Society of Anesthesiology score (2.84 vs. 2.63; $p=0.015$ ) and were more likely to have coronary artery disease $(p<0.001)$, congestive heart failure $(p<0.001)$, or chronic

$\overline{\#}$ These authors contributed equally to this work.

received

April 24, 2018

accepted after revision

April 24, 2019

published online

December 5, 2019
DOI https://doi.org/

$10.1055 / \mathrm{s}-0039-1692456$.

ISSN 2325-4637.
Copyright $\odot 2019$ by Thieme Medical

Publishers, Inc., 333 Seventh Avenue,

New York, NY 10001, USA

Tel: +1(212) 584-4662.
License terms

(1) (1) 
obstructive pulmonary disease $(p<0.001)$. Aneurysm patients were more likely to be former $(p=0.034)$ or current $(p=0.006)$ smokers and had a significantly higher number of pack years $(p<0.001)$. Aneurysm patients also had a significantly poorer lung function. In multivariate analysis, the following factors were associated with aneurysms: chronic obstructive pulmonary disease (odds ratio, $O R=12.24 ; p=0.002$ ), current smoking $(\mathrm{OR}=4.14 ; p=0.002)$, and coronary artery disease $(\mathrm{OR}=2.60$; $p=0.020)$.

Conclusions Our comprehensive analysis identified several comorbidities associated with abdominal aortic aneurysms. These results could help to recognize aneurysms earlier by targeting individuals with these comorbidities for screening.

\section{Introduction}

Abdominal aortic aneurysm (AAA), a dilatation of the infrarenal aorta of $\geq 3 \mathrm{~cm},{ }^{1}$ affects mostly older males. ${ }^{1}$ The age group of 60 to 80 -year-olds is growing, making AAA an increasingly important disease. AAA is often asymptomatic and diagnosed as an incidental abdominal imaging finding. A AAA screening program was introduced in the United States in $2007^{2}$ and in Germany in $2016 .^{3}$

Smoking is the most important risk factor for AAA, increasing the risk for developing an AAA by 7.6 -fold. ${ }^{4}$ Other known risk factors include being male, age $\geq 65$ years, family history for AAA, coronary artery disease (CAD), myocardial infarction, high blood pressure, and chronic obstructive pulmonary disease (COPD). ${ }^{5}$ Protective factors include female sex, Type 2 diabetes, and a black African ethnicity. ${ }^{1}$

Several diseases co-occur with AAA: cholecystolithiasis (CCL), ${ }^{6}$ renal cysts, ${ }^{7}$ COPD, ${ }^{5}$ and hernias ${ }^{8,9}$ are prevalent in the elderly population and share common risk factors with AAA, such as higher age and a positive smoking history. ${ }^{7,10}$

Our goal was to identify additional diseases associated with AAA. They may have similar pathophysiology and could provide a better understanding of the disease, facilitating development of new therapies.

\section{Materials and Methods}

The study was approved by the Charite Ethics Committee (approval number: EA1/309/16).

\section{Study Groups}

Two study groups were created (100/group): (1) AAA group and (2) age- and sex-matched ( \pm 2 years) control group. All 200 individuals had undergone an abdominal computed tomography-angiography (CTA). AAA was defined as the infrarenal diameter of the aorta $\geq 3 \mathrm{~cm} .{ }^{11}$ The AAA patients were operated for AAA from 2004 to 2012 at the Surgical Clinic of Charité Mitte, Berlin, Germany (94 elective and 6 emergency operations). We used the patient's age when diagnosed with AAA, or if this information was missing, the age at the time of AAA operation.

The control patients had no history of AAA surgery, their infrarenal aortic diameter was $<3 \mathrm{~cm}$, and had no other aneurysms. The control patients had received CTA scans at the Department of Radiology, Charité, Berlin, Germany, in 2005 to 2014 either for the evaluation of a kidney donation $(n=14)$ or due to screening for melanoma recurrence $(n=86)$. We used the age at the time of the CTA examination.

\section{Clinical Data}

Details of the variables analyzed are described in the Data Dictionary (-Supplementary Table S1). The information was collected from the medical records and CTA images. We also reviewed findings from abdominal ultrasound examinations, the heart (ejection fraction), and lung function. As FEV1/VC (forced expiratory volume in 1 second in \% of vital capacity) is the most reliable indicator of the exact status of the pulmonary function (restrictive versus obstructive disease), we chose this for the analysis.

The CTA images were evaluated using Centricity eRadCockpit (GE Healthcare, Chalfont St Giles, United Kingdom) for the presence of hernias, diverticulosis, CCL and previously performed cholecystectomy (CCE), and hiatal hernia by two investigators independently. Incisional hernias were excluded from the analysis, as all AAA patients underwent surgery and were exposed to the risk of incisional hernia.

The AAA diameter at initial diagnosis and surgery, graft material used during the operation, and family history were also recorded.

\section{Statistical Analyses}

Analyses were performed using SPSS statistical package (v22 for Windows, IBM, Armonk, NY). First, a univariant comparison of the two groups was made. For quantitative variables, the mean, median, standard deviation, maximum and minimum values were determined. The categorical variables were described by means of cross tabulation. Differences between the two groups were assessed by Mann-Whitney U tests and chi-squared tests (Fisher's exact test). A $p$-value $\leq 0.05$ was considered significant.

To identify the parameters independently associated with AAA, a multivariate analysis was performed and included multiple logistic regression models. Parameters with $>50 \%$ of values missing were not included in the analysis. ORs and $95 \%$ confidence intervals were calculated. 


\section{Results}

The two study groups included 100 AAA and 100 control patients ( 79 men and 21 women/group). The mean age in the AAA group was $69 \pm 8.86$ years, and in the control group $71 \pm 8.59$ years $(p=0.065)$. The average AAA diameter at first contact was $51.78 \pm 14.74 \mathrm{~mm}$ (median: $48 \mathrm{~mm}$; range: 30 $110 \mathrm{~mm}$ ). The AAA diameter at the time of the surgery was $58.76 \pm 12.36 \mathrm{~mm}$ (median: $57 \mathrm{~mm}$ ). The smallest operated aneurysm was $34 \mathrm{~mm}$ in a patient whose rupture-prone aneurysms in the two common iliac arteries required immediate surgery and the small AAA was repaired during the same operation.

Only two (2\%) patients, and none of the controls had a family history of AAA. One AAA patient had died prior to the AAA surgery, and two during hospitalization. Thus, the hospital mortality was $3 \%$. One AAA patient was diagnosed with Marfan syndrome and was excluded from further analyses. There were no differences in the mean height, weight, or body mass index (BMI) between the study groups (-Supplementary Table S2).
AAA patients suffered more frequently from $\operatorname{CAD}(p<0.001)$ and had had a coronary bypass surgery more often $(p=0.007)$, but not coronary stents $(p=0.068)$ ( $($ Table 1$)$. Myocardial infarction $(p=0.035)$ and congestive heart failure $(p<0.001)$ were also more common in the AAA group. AAA patients were significantly more likely to have $\operatorname{PAD}(p=0.037)$. A comparison of the groups, using the Fontaine classification, showed no significant differences between the two groups ( $p=0.157$ ).

COPD was much more common in AAA patients $(p<0.001)$. AAA patients were more likely to be former or current smokers. There was also a highly significant difference $(p<0.001)$ in the mean and median number of pack years between the two groups (mean: 30.57 , median: 25.00 , range: $0-250$ in the AAA group; mean: 10.89 , median: 0, range: 0-60 in the control group). The ex-smokers from the control group had stopped smoking significantly earlier (mean: 14.54, median: 11.00, range: $0-45$ years ago) than the AAA patients (mean: 5.63 , median: 0.00 , range: $0-50$ years ago; $p<0.001$ ).

The echocardiography and pulmonary function results demonstrated significant differences between the AAA and control groups (-Table 2 ).

Table 1 Clinical data gathered from medical records and computed tomography angiography images

\begin{tabular}{|c|c|c|c|c|c|}
\hline \multirow[t]{2}{*}{ Variables } & \multicolumn{2}{|l|}{ AAA } & \multicolumn{2}{|l|}{ Control } & \multirow[t]{2}{*}{$p$-Value ${ }^{a}$} \\
\hline & $\begin{array}{l}\text { With } \\
\text { variable, } n\end{array}$ & $\begin{array}{l}\text { Total with } \\
\text { data, } n\end{array}$ & $\begin{array}{l}\text { With } \\
\text { variable, } n\end{array}$ & $\begin{array}{l}\text { Total with } \\
\text { data, } n\end{array}$ & \\
\hline Hypertension & 78 & 98 & 70 & 100 & 0.142 \\
\hline Coronary artery disease & 49 & 98 & 19 & 100 & $<0.001$ \\
\hline Coronary bypass & 17 & 99 & 5 & 100 & 0.007 \\
\hline PTCA & 23 & 99 & 13 & 100 & 0.068 \\
\hline Myocardial infarction & 26 & 99 & 14 & 100 & 0.035 \\
\hline Peripheral artery disease & 19 & 99 & 8 & 99 & 0.037 \\
\hline $\begin{array}{l}\text { Peripheral artery disease stage: } \\
\text { Ila/IIb/III/IV/bypass/PTA }\end{array}$ & $5 / 7 / 2 / 2 / 3 / 0$ & 99 & $2 / 1 / 1 / 1 / 2 / 1$ & 99 & 0.157 \\
\hline Atrial fibrillation & 14 & 99 & 10 & 100 & 0.497 \\
\hline Artificial pacemaker & 2 & 99 & 1 & 100 & 0.497 \\
\hline TIA & 6 & 99 & 3 & 100 & 0.323 \\
\hline Stroke & 9 & 99 & 5 & 100 & 0.323 \\
\hline Congestive heart failure, all & 20 & 99 & 2 & 99 & $<0.001$ \\
\hline $\begin{array}{l}\text { Congestive heart failure classes: } \\
\text { NYHA } 1 / 2 / 3 / 4\end{array}$ & $5 / 7 / 7 / 1$ & 99 & $0 / 2 / 0 / 0$ & 99 & $<0.001$ \\
\hline Diabetes mellitus, all & 18 & 99 & 19 & 97 & 0.856 \\
\hline Diabetes mellitus: diet/medication/insulin & $10 / 6 / 2$ & 99 & $0 / 14 / 5$ & 97 & 0.001 \\
\hline Hyperlipidemia & 35 & 98 & 34 & 98 & 1.000 \\
\hline COPD & 28 & 98 & 4 & 100 & $<0.001$ \\
\hline Ever-smoker & 65 & 94 & 46 & 87 & 0.034 \\
\hline Current smoker & 32 & 95 & 13 & 86 & 0.006 \\
\hline Hypothyroidism & 10 & 99 & 6 & 100 & 0.311 \\
\hline
\end{tabular}

Abbreviations: AAA, abdominal aortic aneurysm; COPD, chronic obstructive pulmonary disease; NYHA, New York Heart Association; PTA, percutaneous transluminal angioplasty; PTCA, percutaneous transluminal coronary angioplasty; TIA, transient ischemic attack.

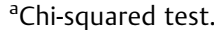

Note: Refer Data Dictionary in - Supplementary Table S1 for definitions of variables. 
Table 2 Ejection fraction and pulmonary function

\begin{tabular}{|l|l|l|l|l|l|l|l|}
\hline Variables & \multicolumn{2}{|l|}{ AAA } & \multicolumn{2}{l|}{ Control } & $p_{\text {-Value }}$ \\
\cline { 2 - 8 } & Mean \pm SD & Median & $\begin{array}{l}\text { Data } \\
\text { available, } \boldsymbol{n}\end{array}$ & Mean \pm SD & Median & $\begin{array}{l}\text { Data } \\
\text { available, } \mathbf{n}\end{array}$ \\
\hline Ejection fraction (\%) & $54.04 \pm 11.69$ & 60 & $66^{\mathrm{b}}$ & $59.27 \pm 6.02$ & 60 & $26^{\mathrm{c}}$ & 0.075 \\
\hline FEV1 in \% VC & $66.85 \pm 16.00$ & 71 & $58^{\mathrm{d}}$ & $78.17 \pm 8.82$ & 80 & $21^{\mathrm{e}}$ & $\mathbf{0 . 0 0 3}$ \\
\hline
\end{tabular}

Abbreviations: AAA, abdominal aortic aneurysm; FEV1, forced expiratory volume in one second; SD, standard deviation; VC, vital capacity. ${ }^{a}$ Mann-Whitney $U$ test.

${ }^{\mathrm{b}}$ Mean age $69 \pm 8.99,55$ men, 11 women.

${ }^{c}$ Mean age $68 \pm 10.08,21$ men, 5 women.

${ }^{\mathrm{d}}$ Mean age $67 \pm 8.55,48$ men, 10 women.

${ }^{\mathrm{e}}$ Mean age $65 \pm 10.98,18$ men, 3 women.

Table 3 Presence of cholecystolithiasis, diverticulosis, pancreatic, and splenic cysts based on computed tomography-angiography imaging data

\begin{tabular}{|l|l|l|l|}
\hline Variables & $\begin{array}{l}\text { AAA } \\
(\boldsymbol{n}=\mathbf{9 9}\end{array}$ & $\begin{array}{l}\text { Control } \\
(\boldsymbol{n}=\mathbf{1 0 0})\end{array}$ & p-Value \\
\hline CCL, ever & 27 & 29 & 0.875 \\
\hline CCL, present & 12 & 10 & 0.743 \\
\hline Cholecystectomy & 15 & 19 & 0.743 \\
\hline Diverticulosis & 44 & 31 & $\mathbf{0 . 0 0 6}$ \\
\hline Diverticulitis & 4 & 0 & $\mathbf{0 . 0 0 6}$ \\
\hline Sigma resection & 1 & 0 & $\mathbf{0 . 0 0 6}$ \\
\hline Pancreatic cysts & 1 & 0 & 0.497 \\
\hline Splenic cysts & 1 & 3 & 0.621 \\
\hline
\end{tabular}

Abbreviations: AAA, abdominal aortic aneurysm; CCL, cholecystolithiasis.

Note: Computed tomography-angiography images were analyzed for 199 study patients. AAA patient with Marfan syndrome was excluded from the analysis.

${ }^{a}$ Chi-squared test.

Table 4 Presence of inguinal and umbilical hernia

\begin{tabular}{|l|l|l|l|}
\hline & $\begin{array}{l}\text { AAA } \\
(\boldsymbol{n}=\mathbf{9 9})\end{array}$ & $\begin{array}{l}\text { Control } \\
(\boldsymbol{n}=\mathbf{1 0 0})\end{array}$ & -Value $^{\mathrm{a}}$ \\
\hline Hernia, ever $^{\mathrm{b}}$ & $19^{\mathrm{c}}$ & 10 & 0.073 \\
\hline Inguinal hernia, ever & 16 & 9 & 0.140 \\
\hline Inguinal hernia, present & 5 & 4 & 0.280 \\
\hline $\begin{array}{l}\text { Inguinal hernia, surgical } \\
\text { repair }\end{array}$ & 11 & 5 & 0.280 \\
\hline Umbilical hernia, ever & 4 & 1 & 0.212 \\
\hline
\end{tabular}

Abbreviations: AAA, abdominal aortic aneurysm.

Note: Computed tomography-angiography images were analyzed for 199 study patients. The AAA patient with Marfan syndrome was excluded from the analysis.

${ }^{a}$ Chi-squared test.

${ }^{b}$ All patients with a hernia diagnosis in their medical history.

'One patient had two types of hernia: inguinal and umbilical hernia.

No significant difference was found in the frequency of CCL ( - Table 3 ) or hernias ( - Table 4) between the two groups. Diverticulosis was diagnosed significantly more often in the AAA group $(p=0.006 ;-$ Table 3$)$. Five hiatal hernias were diagnosed in both groups $(p=1.000)$. A splenic cyst was found in one AAA patient and three control patients $(p=0.621)$. One AAA patient had a pancreatic cyst.

The main differences in laboratory results were lower platelet count $(p=0.035)$ and higher creatinine levels $(p=0.009)$ in the AAA group ( - Table 5$)$. The AAA patients also had significantly higher thyroid-stimulating hormone levels ( $p=0.032$ ). There were no differences in the ASA scores (-Supplementary Table S3), blood groups (-Supplementary Table 54), or Rhesus factor distributions (-Supplementary Table S5).

Results of the multivariate analysis showed the strongest association between COPD and AAA $(\mathrm{OR}=12.242$; $p=0.002)$. Current smoking ( $\mathrm{OR}=4.141 ; p=0.002)$ and $\mathrm{CAD}(\mathrm{OR}=2.603 ; p=0.020)$ also had a strong association with AAA (-Table 6). There was no direct association between $A A A$ and the number of pack years $(O R=1.015$; $p=0.275)$ or ever-smoking ( $\mathrm{OR}=1.488 ; p=0.575)$. Diverticulosis showed a borderline association with AAA (OR $=1.844 ; p=0.075$ ). Platelet count revealed an indirect association with $\mathrm{AAA}(\mathrm{OR}=0.994 ; p=0.023)$.

\section{Discussion}

We found that COPD, CAD, congestive heart failure, and PAD were significantly more common among AAA patients than age- and sex-matched controls. The AAA patients received more coronary bypass implants, but not coronary stents. The control patients had a significantly higher FEV1/VC. Diverticulosis was observed significantly more frequently in AAA patients.

We identified COPD as the strongest independently associated disease with AAA, which is in agreement with previous studies reporting a prevalence of COPD up to $44 \%$ among AAA patients. ${ }^{5}$ The FEV1 value is strongly associated with COPD. It is not surprising that the AAA patients had a significantly lower FEV1/VC ratio than controls (67 vs. $78 \%$ ), a result comparable to other studies. ${ }^{12}$ These findings are clinically important, especially for the surgical repair of AAA, since COPD is also a known risk factor for a poor outcome after a major surgery, ${ }^{1}$ making it important to control COPD medically before a planned AAA surgery. Cronenwett et $\mathrm{al}^{13}$ showed that the presence of COPD increases the risk of AAA rupture. 
Table 5 Comparison of laboratory data between abdominal aortic aneurysm and control group

\begin{tabular}{|c|c|c|c|c|c|c|c|}
\hline \multirow[t]{2}{*}{ Variable } & \multicolumn{3}{|l|}{ AAA } & \multicolumn{3}{|l|}{ Control } & \multirow[t]{2}{*}{$p$-Value } \\
\hline & Mean \pm SD & Media, $n$ & $\begin{array}{l}\text { Data } \\
\text { available, } n\end{array}$ & Mean \pm SD & Median & $\begin{array}{l}\text { Data } \\
\text { available, } n\end{array}$ & \\
\hline Hemoglobin & $13.75 \pm 1.95$ & 13.85 & 98 & $13.29 \pm 2.01$ & 13.40 & 97 & 0.164 \\
\hline Hematocrit & $0.41 \pm 0.054$ & 0.42 & 99 & $0.398 \pm 0.054$ & 0.41 & 97 & 0.188 \\
\hline Leukocytes & $7.99 \pm 3.09$ & 7.23 & 99 & $8.67 \pm 6.13$ & 7.13 & 97 & 0.540 \\
\hline Platelet count & $220.22 \pm 58.41$ & 211 & 99 & $253.51 \pm 94.97$ & 238 & 97 & 0.035 \\
\hline Glucose & $115.12 \pm 36.22$ & 104 & 68 & $110.92 \pm 43.56$ & 96 & 51 & 0.092 \\
\hline CRP & $14.21 \pm 20.06$ & 5.85 & 54 & $29.31 \pm 54.14$ & 3.85 & 76 & 0.455 \\
\hline Creatinine & $1.33 \pm 1.04$ & 1.09 & 97 & $1.04 \pm 0.39$ & 0.93 & 97 & 0.009 \\
\hline $\mathrm{TSH}$ & $2.12 \pm 3.47$ & 1.39 & 47 & $1.36 \pm 1.22$ & 1.13 & 75 & 0.032 \\
\hline
\end{tabular}

Abbreviations: AAA, abdominal aortic aneurysm; CRP, C-reactive protein; SD, standard deviation; TSH, thyroid-stimulating hormone.

Table 6 Results of multivariate analysis after forward and backward selection

\begin{tabular}{|l|l|l|l|l|}
\hline Parameter & OR & $95 \%$ Confidence interval & $n$ & $p$-Value \\
\hline Ever smoker & 1.488 & $0.371-5.960$ & 123 & 0.575 \\
\hline PAD & 0.979 & $0.566-1.693$ & 123 & 0.938 \\
\hline Pack years & 1.015 & $0.988-1.042$ & 123 & 0.275 \\
\hline Congestive heart failure & 2.408 & $0.352-16.468$ & 123 & 0.370 \\
\hline ASA score & 0.810 & $0.373-2,160$ & 123 & 0.898 \\
\hline Diabetes mellitus & 0.950 & $0.491-1.835$ & 123 & 0.878 \\
\hline Coronary bypass & 1.106 & $0.132-9.287$ & 123 & 0.926 \\
\hline Creatinine & 1.037 & $0.245-4.388$ & 123 & 0.961 \\
\hline COPD & 12.242 & $2.584-57.998$ & 180 & $\mathbf{0 . 0 0 2}$ \\
\hline Current smoker & 4.141 & $1.719-9.977$ & 180 & $\mathbf{0 . 0 0 2}$ \\
\hline Coronary artery disease & 2.603 & $1.160-5.838$ & 180 & $\mathbf{0 . 0 2 0}$ \\
\hline Diverticulosis & 1.844 & $0.941-3.615$ & 180 & 0.075 \\
\hline Platelet count & 0.994 & $0.989-0.999$ & 180 & $\mathbf{0 . 0 2 3}$ \\
\hline
\end{tabular}

Abbreviations: ASA, American Society of Anesthesiologists; COPD, chronic obstructive pulmonary disease; OR, odds ratio; PAD, peripheral arterial disease.

We found a strong association with AAA and cardiovascular diseases including CAD, congestive heart failure, and PAD. Some previous studies, ${ }^{10}$ but not all, ${ }^{14}$ showed an association between AAA and CAD. Several studies also mentioned an association between AAA and myocardial infarction, ${ }^{15}$ which was confirmed in our study. Our study showed a significantly higher prevalence of congestive heart failure in the AAA group ( 20 vs. $2 \%$ ), but no direct association with AAA in the multivariate analysis. One previous study found a prevalence of congestive heart failure to be $24 \%$ among AAA patients, ${ }^{16}$ which is similar to our results.

Smoking is a well-established risk factor for AAA, ${ }^{1,12,17}$ a finding confirmed in our study $(\mathrm{OR}=4.141)$. The risk increases with the number of pack years, and even after stopping smoking, the increased risk remains apparent for decades. ${ }^{17}$ Current smoking increases both the expansion and the rupture rate of AAA, as well as perioperative mortality. ${ }^{17}$
Some studies, ${ }^{8}$ but not all, ${ }^{18}$ describe an association of hernias and AAA. Our study demonstrated higher prevalence of all hernias in the AAA (19\%) than in the control group (10\%); however, the difference was not significant. Alnassar et $\mathrm{al}^{19}$ reported that $1.3 \%$ of AAA patients were diagnosed with an umbilical hernia, whereas in our study $4 \%$ of AAA and $1 \%$ of the control patients had an umbilical hernia. In both diseases, the inflammatory process is a prominent feature, ${ }^{8}$ and mainly older men with a family history of smoking and COPD are affected. ${ }^{9}$

AAA and CCL have rarely been examined together. We found a $12 \%$ prevalence of CCL among the AAA patients, which is slightly higher than most previous studies have reported (4.9$11 \%){ }^{20,21}$ Schuster et $\mathrm{al}^{6}$ revealed a significantly higher prevalence of CCL and CCE among AAA (50 and 11\%, respectively) compared with controls ( 26 and $7 \%$, respectively) and demonstrated an association between AAA and CCL in multivariate analysis. Our study could not confirm this finding. 
In our study, $31 \%$ of control and $50 \%$ of AAA patients had a diagnosis of diverticulosis, but there was no direct association between diverticulosis and AAA in multivariable analyses. The prevalence of diverticulosis in the general population is 20 to $30 \%$ and rises sharply with older age. ${ }^{22}$ Results of our study are, therefore, similar to the literature. Only one study has described an independent association between AAA and diverticulosis. ${ }^{23}$ The Saint's Triad is thought to be a manifestation of a general connective tissue disorder, resulting in the formation of hernias, diverticula, aneurysms, or COPD. ${ }^{23}$ If a diverticulitis develops, a diverticulum is more likely to perforate. In case of a free perforation, a peritonitis will occur and immediate colon surgery is necessary.

\section{Limitations}

Limitations of our study are as follows: (1) It was a retrospective, single-center study of 100 AAA and 100 control patients. (2) Because the patient data were obtained from medical records and CT images, there is a possibility the data were incomplete. (3) The control group (kidney donation and melanoma patients) may not be representative of the general population. (4) We only compared operated AAA patients and the results presented here are, therefore, relevant for large AAAs.

Regarding the control group in our study, we would like to note that the findings agree with those from previous studies in which the control group consisted of patients who underwent thoracic and cardiovascular surgery ${ }^{7}$ or had aortoiliac occlusive disease. ${ }^{24}$ For example, Pitoulias et $\mathrm{al}^{24}$ showed a significantly higher prevalence of COPD in the AAA study population as was seen in the current study. It could be argued that the melanoma patients formed an appropriate control group, since melanoma is an age-related disease and a disease of a different organ, not the aorta. There were no differences in the mean height, weight, or BMI between the AAA and control groups as shown in - Supplementary Table S2.

An advantage of our study was the matching of study groups for age and sex. This was all the more important because many of the diseases studied (e.g., CCL, diverticulosis, and COPD) are more common in the elderly population. Matching could also reduce the sex-related differences, since male sex is a known risk factor for AAA, while female sex is a risk factor for the CCL.

\section{Conclusions}

This study provides a comprehensive characterization of the clinical picture of AAA and its associated comorbidities. The findings have several clinical implications emphasizing the need for a better risk stratification of AAA patients, which could lead to earlier detection of AAA. Screening programs for patients at risk could be created, and a cost-benefit analysis performed, especially among older men with a history of smoking. Other risk factors (e.g., COPD) should be taken into consideration. Our study confirmed that AAA patients often suffer from serious secondary diseases such as COPD, CAD, or heart failure. These are often the reasons for a poor postoperative outcome and increased mortality. Less serious diseases such as CCL and diverticulosis should not be forgotten. Comprehensive knowledge about AAA and its comorbidities facilitates future development of therapies.

Funding

None.

\section{Conflict of Interest}

The authors declare no conflict of interest related to this article.

\section{Acknowledgment}

The authors would like to acknowledge support from the German Research Foundation (DFG) and the Open Access Publication Funds of Charité - Universitätsmedizin, Berlin.

\section{References}

1 Moll FL, Powell JT, Fraedrich G, et al; European Society for Vascular Surgery. Management of abdominal aortic aneurysms clinical practice guidelines of the European Society for Vascular Surgery. Eur J Vasc Endovasc Surg 2011;41(Suppl 1):S1-S58

2 Lederle FA. Screening for AAA in the USA. Scand J Surg 2008;97 (02):139-141

3 Medizin S. Bauchaorten-Screening bald im Check-up. MMW Fortschr Med 2016;158:33. Doi: 10.1007/s15006-016-8991-5

4 Nordon IM, Hinchliffe RJ, Loftus IM, Thompson MM. Pathophysiology and epidemiology of abdominal aortic aneurysms. Nat Rev Cardiol 2011;8(02):92-102

5 Meijer CA, Kokje VB, van Tongeren RB, et al. An association between chronic obstructive pulmonary disease and abdominal aortic aneurysm beyond smoking: results from a case-control study. Eur J Vasc Endovasc Surg 2012;44(02):153-157

6 Schuster JJ, Raptopoulos V, Baker SP. Increased prevalence of cholelithiasis in patients with abdominal aortic aneurysm: sonographic evaluation. AJR Am J Roentgenol 1989;152(03):509-511

7 Ito T, Kawaharada N, Kurimoto Y, et al. Renal cysts as strongest association with abdominal aortic aneurysm in elderly. Ann Vasc Dis 2010;3(02):111-116

8 Antoniou GA, Georgiadis GS, Antoniou SA, Granderath FA, Giannoukas AD, Lazarides MK. Abdominal aortic aneurysm and abdominal wall hernia as manifestations of a connective tissue disorder. J Vasc Surg 2011;54(04):1175-1181

9 Antoniou GA, Giannoukas AD, Georgiadis GS, et al. Increased prevalence of abdominal aortic aneurysm in patients undergoing inguinal hernia repair compared with patients without hernia receiving aneurysm screening. J Vasc Surg 2011;53(05):1184-1188

10 Chun KC, Teng KY, Chavez LA, et al. Risk factors associated with the diagnosis of abdominal aortic aneurysm in patients screened at a regional veterans affairs health care system. Ann Vasc Surg 2014;28(01):87-92

11 Johnston KW, Rutherford RB, Tilson MD, Shah DM, Hollier L, Stanley JC. Suggested standards for reporting on arterial aneurysms. Subcommittee on Reporting Standards for Arterial Aneurysms, Ad Hoc Committee on Reporting Standards, Society for Vascular Surgery and North American Chapter, International Society for Cardiovascular Surgery. JVasc Surg 1991;13(03): 452-458

12 Fowkes FG, Anandan CL, Lee AJ, et al. Reduced lung function in patients with abdominal aortic aneurysm is associated with activation of inflammation and hemostasis, not smoking or cardiovascular disease. J Vasc Surg 2006;43(03):474-480

13 Cronenwett JL, Murphy TF, Zelenock GB, et al. Actuarial analysis of variables associated with rupture of small abdominal aortic aneurysms. Surgery 1985;98(03):472-483 
14 Spanos K, Rountas C, Saleptsis V, Athanasoulas A, Fezoulidis I, Giannoukas AD. The association of simple renal cysts with abdominal aortic aneurysms and their impact on renal function after endovascular aneurysm repair. Vascular 2016;24(02): 150-156

15 Cowan JAJr, Dimick JB, Henke PK, Rectenwald J, Stanley JC, Upchurch GR Jr. Epidemiology of aortic aneurysm repair in the United States from 1993 to 2003. Ann N Y Acad Sci 2006;1085:1-10

16 Barisione C, Garibaldi S, Brunelli C, et al. Prevalent cardiac, renal and cardiorenal damage in patients with advanced abdominal aortic aneurysms. Intern Emerg Med 2016;11(02):205-212

17 Norman PE, Curci JA. Understanding the effects of tobacco smoke on the pathogenesis of aortic aneurysm. Arterioscler Thromb Vasc Biol 2013;33(07):1473-1477

18 Henriksen NA, Sorensen LT, Jorgensen LN, Lindholt JS. Lack of association between inguinal hernia and abdominal aortic aneurysm in a population-based male cohort. Br J Surg 2013;100(11): $1478-1482$
19 Alnassar S, Bawahab M, Abdoh A, Guzman R, Al Tuwaijiri T, Louridas G. Incisional hernia postrepair of abdominal aortic occlusive and aneurysmal disease: five-year incidence. Vascular 2012;20(05):273-277

20 Ouriel K, Ricotta JJ, Adams JT, Deweese JA. Management of cholelithiasis in patients with abdominal aortic aneurysm. Ann Surg 1983;198(06):717-719

21 Thomas JH. Abdominal aortic aneurysmorrhaphy combined with biliary or gastrointestinal surgery. Surg Clin North Am 1989;69 (04):807-815

22 Bogardus ST Jr. What do we know about diverticular disease? A brief overview. J Clin Gastroenterol 2006;40(Suppl 3):S108-S111

23 Hauer-Jensen M, Bursac Z, Read RC. Is herniosis the single etiology of Saint's triad? Hernia 2009;13(01):29-34

24 Pitoulias GA, Donas KP, Chatzimavroudis G, Torsello G, Papadimitriou DK. The role of simple renal cysts, abdominal wall hernia, and chronic obstructive pulmonary disease as predictive factors for aortoiliac aneurysmatic disease. World J Surg 2012;36(08): 1953-1957 